\title{
Evaluation of Empirical Antibiotic Treatment in Culture Negative Pyogenic Vertebral Osteomyelitis
}

\author{
Mustafa DOGAN ${ }^{1}$, Abdullah Talha SIMSEK ${ }^{2}$, Ibrahim YILMAZ3,Numan KARAARSLAN ${ }^{4}$ \\ ${ }^{1}$ Namik Kemal University School of Medicine, Department of Infectious Diseases, Tekirdag, Turkey \\ ${ }^{2}$ Harakani State Hospital, Department of Neurosurgery, Kars, Turkey \\ ${ }^{3}$ Istanbul Medipol University School of Medicine, Department of Medical Pharmacology, Istanbul, Turkey \\ ${ }^{4}$ Namik Kemal University School of Medicine, Department of Neurosurgery, Tekirdag, Turkey
}

Corresponding author: Numan KARAARSLAN numikara@yahoo.com

\section{ABSTRACT}

AIM: To investigate the efficacy of empirical antibiotic treatment in culture-negative pyogenic vertebral osteomyelitis (PVO) cases.

MATERIAL and METHODS: The records of patients with culture-negative PVO who were treated at infectious diseases and neurosurgery outpatient clinics in the past four years were examined retrospectively. The control group comprised healthy subjects with similar age, gender, and body mass index but without pathology. The comparison of the groups was performed by analysis of variance. Statistical significance was accepted as $p<0.05$.

RESULTS: No statistically significant difference in the white blood cell count and erythrocyte sedimentation rate was found between the spondylodiscitis and the healthy subject groups when the blood parameters obtained before and after the treatment ( $p>0.05)$. However, a statistical significance was assessed in the results of the comparison for C-reactive protein $(p<0.05)$.

CONCLUSION: In the context of evidence-based medicine and the rational use of antibiotics, it is clear that antibiotics should be preferred according to the culture antibiogram results in the treatment of infectious diseases.

KEYWORDS: Empirical treatment, Pyogenic vertebral osteomyelitis, Rational antibiotic use, Rational drug use, Spondylodiscitis

\section{INTRODUCTION}

$\mathrm{P}$ yogenic vertebral osteomyelitis (PVO), also called spondylodiscitis, spondylitis, or disc area infection, is a rare pathologic disorder that may cause significant problems in its diagnosis and treatment. This pathology is a clinical condition that can affect the epidural space, muscle, fascia, and adjacent soft tissues due to the local spread of the infection (19).

It has been suggested that the increase in the incidence of PVO in recent years may be due to the increased availability of more sensitive diagnostic methods (9). Identification of the active microorganism is important in determining the appropriate antibiotic treatment option. However, microbiological factors have not yet been determined even though many methods, such as surgical or needle aspiration biopsy, wound swab sampling, and taking a blood culture, have been used $(7,14)$. The ideal diagnostic method is not yet fully evident. Furthermore, the literature shows there is an increase in the culture-negative PVO rate (14).

The World Health Organization emphasizes that patients should use the medications that are appropriate to their clinical needs, in doses that meet their own individual requirements, for an adequate period, and at the lowest cost to them and their community $(10,16,20)$. The application of drugs, also called "rational antibiotic use", necessitates the development of appropriate antibiotic usage policies. Such policies are very important for patient survival, prevention of complications and chronicity, and shortening disease severity and duration (11).
Mustafa DOGAN $\quad$ (1) : 0000-0002-3341-925X Abdullah Talha SIMSEK (D): 0000-0002-8668-3935
Ibrahim YILMAZ OD: :0000-0003-2003-6337

Numan KARAARSLAN (D) : 0000-0001-5590-0637 
However, the early diagnosis and treatment of PVO remain major problems despite the progress in surgical treatment and diagnostic interventions. In addition, despite the range of antibiotic options that is available, successful treatment in PVO cases is limited. As a result, the long-term hospitalization and medication of patients continue to be major problems. In addition, the most appropriate antibiotic usage, its dosage, and treatment period in these patients remain separate problems (16).

This study was carried out to investigate empirical antibiotic use, including its treatment efficacy and compliance of treatment options with the principles of rational antibiotic use in culture-negative PVO cases. Such cases may be encountered in neurosurgery and infectious diseases practices, where the active agent might not be detected with the help of current serological and microbiological diagnostic methods.

\section{MATERIAL and METHODS}

The data from both patients and healthy volunteers were obtained from the hospital information management system databases. Approval to use the relevant data was obtained from the hospital administration (12.04.2018/67087600).

In this retrospective controlled study, the records of patients admitted to the infectious disease and neurosurgery clinic with increasing back pain between January 2015 and January 2018 were examined. The patients had undergone physical and neurological examinations, including conventional radiological imaging and magnetic resonance imaging (MRI). Patients diagnosed with PVO $(n=24)$ were selected for the study and their data were analyzed.

\section{Materials}

The commercial kits used to perform the Brucella agglutination test and the Wright-Brucella tube agglutination tests were (Cat $\neq 190131)$.

\section{Study Design}

The data of the patients diagnosed with PVO were obtained by screening the record archive. The cases with negative results for the Rose Bengal Brucella slide agglutination and Wright Brucella tube agglutination tests were included in the study.

In addition, the study also included the cases in which tuberculosis and non-tuberculosis mycobacterial infections, fungal infections, and Brucella were not detected after examining the culture tests of the specimens taken by aspiration or open biopsy, and which also met the inclusion criteria for the research.

Microbiological analyses of the samples taken for diagnostic purposes were performed. Values of white blood cells (WBC), C-reactive protein (CRP), and erythrocyte sedimentation rate (ESR) were recorded before and after empirical antibiotherapy. The data were recorded in a newly created standard form. In addition, all the data about the antibiotics that were prescribed and administered to patients with PVO during hospitalization and following discharge were recorded.
To evaluate the results of antibiotherapy applied to these patients, a control group was formed. For this purpose, laboratory data of the healthy volunteers $(n=24)$, who were similar in terms of body mass index, age and gender, and referred to the neurosurgery clinic, were used. This group was the control group.

To test the efficacy of empirical antibiotherapy in the patients in the study group, the CRP, ESR, and WBC values of the study group patients were compared to those in the control group.

In addition, to evaluate the efficacy of antibiotic therapy, the spinal MRIs of the patients in the study group, performed before the treatment and six months after the termination of the treatment, were compared (Figure 1A-R).

\section{Eligibility Criteria}

Patient demographic data, comorbidities, coexisting infections, level of spine involvement, pre-treatment neurological status, and prior antibiotic therapy were documented.

After the file archive scans, the presence of rheumatoid diseases and malignancies that may increase ESR and CRP levels were observed and taken into consideration in the study group patients (6).

The criteria used by Beronius et al. for the diagnosis of spondylodiscitis were used in the diagnosis of PVO (4). Four patients who were initially included in the study $(n=24)$ were excluded when other issues were found, such as malignancy $(n=1)$, rheumatoid disease $(n=1)$ and those treated for another infection at that time $(n=2)$.

Of the 20 patients that satisfied the inclusion criteria, 8 patients were diagnosed with PVO without a causative agent after microbiological examination of samples obtained through aspiration and open biopsy. Evaluations were performed on these patients.

\section{Statistical Analyses}

The statistical analyses were performed using Minitab software (version 18.0) and the data were evaluated at a confidence interval of $95 \%$. Descriptive statistics were presented as the mean \pm standard deviation (SD), frequency (\%), and standard error of the mean (SEM). The difference in the significance between the averages of the groups was tested using the F-test analysis of variance (ANOVA). A two-sample independent t-test, which compares two sample groups, was performed to determine whether there was a significant difference in the efficacy of the antibiotics administered to the spondylodiscitis group before the treatment. The alpha significance value was accepted as $p<0.05$.

\section{RESULTS}

The control group comprised 14 male subjects and 10 female subjects. Fifty percent of the patients in the PVO group were female (Table I).

The biopsy samples of the patients in the study group were taken through percutaneous needle biopsy with guided imaging and open surgery (Table II). 

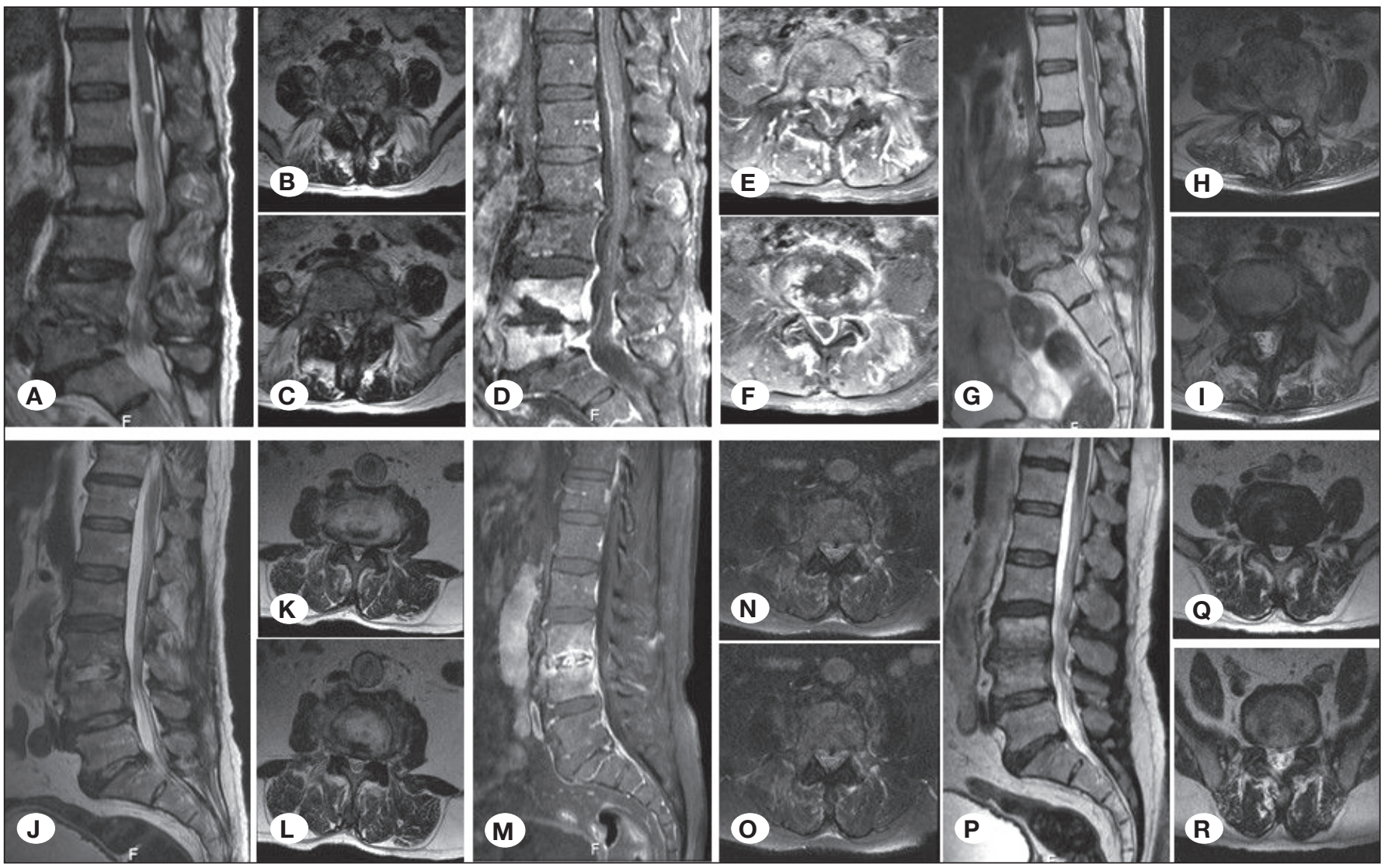

Figure 1: Magnetic resonance imaging (MRI) sections before and after antibiotherapy in two different pyogenic vertebral osteomyelitis (PVO) cases. A-F) The first case's pretreatment contrast-enhanced and non-contrast T2W sagittal and axial MRI sections revealed the PVO where L4-5 levels are affected. J-O) The other case's pretreatment contrast-enhanced and non-contrast T2W sagittal and axial MRI sections revealed PVO where L3-4 levels are affected. G, H, I, P, Q, and R: non-contrast T2W sagittal and axial MRI sections of both cases six months after antibiotic administration.

Table I: Comparison of Demographic Data and Peripheral Vein Sampling Parameters Between Groups.

\begin{tabular}{lccc}
\hline & $\begin{array}{c}\text { Control group } \\
\text { (Healthy Volunteer) } \\
(\mathbf{n}=\mathbf{2 4})\end{array}$ & $\begin{array}{c}\text { Study group (Spondylodiscitis) } \\
\text { Before Antibioterapy } \\
(\mathbf{n}=\mathbf{8})\end{array}$ & $\begin{array}{c}\text { Study group (Spondylodiscitis) } \\
\text { After Antibioterapy } \\
(\mathbf{n}=\mathbf{8})\end{array}$ \\
\hline Age (year) & $46.38 \pm 12.76$ & $48.56 \pm 11.44$ & $48.56 \pm 11.44$ \\
\hline WBC $(\mathrm{e} 3 / \mathrm{UL})$ & $8.36 \pm 2.78$ & $9.78 \pm 3.68$ & $7.18 \pm 1.52$ \\
\hline $\mathrm{ESR}(\mathrm{mm} / \mathrm{H})$ & $11.5 \pm 12.37$ & $70.25 \pm 22.96$ & $41.5 \pm 30.09$ \\
\hline $\mathrm{CRP}(\mathrm{mg} / \mathrm{dL})$ & $3.49 \pm 1.89$ & $95.19 \pm 84.17$ & $15.03 \pm 23.58$ \\
\hline
\end{tabular}

ESR: Erythrocyte sedimentation rate, WBC: white blood cell, CRP: C-reactive protein.

Table II: Culture Samples and Gram Staining Results of the Spondylodiscitis Group

\begin{tabular}{lcc}
\hline Application & Pathogen & Frequency (\%) \\
\hline Open Biopsy & No & 17.39 \\
\hline Percutaneous Biopsy & No & 8.7 \\
\hline Wound Swab & No & 8.7 \\
\hline
\end{tabular}

It was observed that $33.33 \%$ empirical antibiotic treatment was applied to the patients without microorganism $(n=8)$. The mean duration of intravenous antibiotherapy was $48.63 \pm$ 36.18 (SD) days (up to 105 days, minimum 28 days).

After the patients were hospitalized, the antibiotics having cefazolin, ampicillin/sulbactam, piperacillin/tazobactam, linezolid, and clindamycin as their active substance were planned to be administered intravenously, either mono or in combination. 
When the clinical records were examined, it was observed that the amoxicillin/clavulanic acid, ciprofloxacin, rifampicin, linezolid, and clindamycin active antibiotics were prescribed to the patients for a period of at least two weeks after the clinical symptoms regressed.

After patients' files were scanned in the archive file, the cessation of antibiotic use was observed to be recommended following regression in CRP and ESR values (laboratory parameters related to infection) toward normal values, on the advice of the infectious disease physician.

No statistically significant difference was found in terms of gender between the control group and the patients in the spondylodiscitis group $(p>0.05)$. However, there was a statistically significant difference in the CRP values $(f=6.01$, $\mathrm{p}=0.03$ ) (Figure 2).

Despite the different antibiotics applied to three patients in the study group, the response to this treatment was limited. These patients underwent surgical debridement and abscess aspiration.

There was no statistically significant difference (WBCT: 0.96, $\mathrm{p}=0.351$; ESRT: $-2.10, \mathrm{p}=0.069$; CRPT:-1.45, $\mathrm{p}=0.190$ ) between the blood parameters obtained from healthy volunteers and from the spondylodiscitis group before and after treatment $(p>0.05)$.

\section{DISCUSSION}

Antibiotic resistance has become an important health problem around the world. Optimal antibiotic usage requires proper administration of the appropriate antibiotic at the appropriate time. The presence of a microbiologically proven bacterial infection must be investigated for the right antibiotic usage. The improper use of antibiotics refers to cases in which antibiotics are used without the presence of proven infection, where inefficient antibiotics or several antibiotics are used in the presence of infection, or where the antibiotic dosages and their usage intervals are inappropriate. This causes an increase in bacterial resistance to the drugs. In addition, this may result in the prolongation of hospital stay and loss of labor, treatment failures, adverse drug reactions, side effects, increased treatment costs, and mortality $(2,3,10,20)$.

In this study, the efficacy of empirical antibiotic use in treatment and its compliance with the principles of rational use of antibiotics were evaluated in light of the literature regarding patients diagnosed with PVO. These patients had biopsies for diagnostic purposes and they had no proliferation, according to the culture antibiogram result.

In the literature, it has been reported that the empirical therapies are useful in the treatment of spondylodiscitis $(1,5,18)$. However, it has also been reported that spondylodiscitis cannot be treated with empirical intravenous antibiotics (13).

In their research, Cervan et al. focused on the results of the diagnosis and treatment of 23 patients with spondylodiscitis under hemodialysis treatment (5). They emphasized that early negative cultures were not uncommon $(n=11)$ in these patients and that they benefited from MRI in addition to

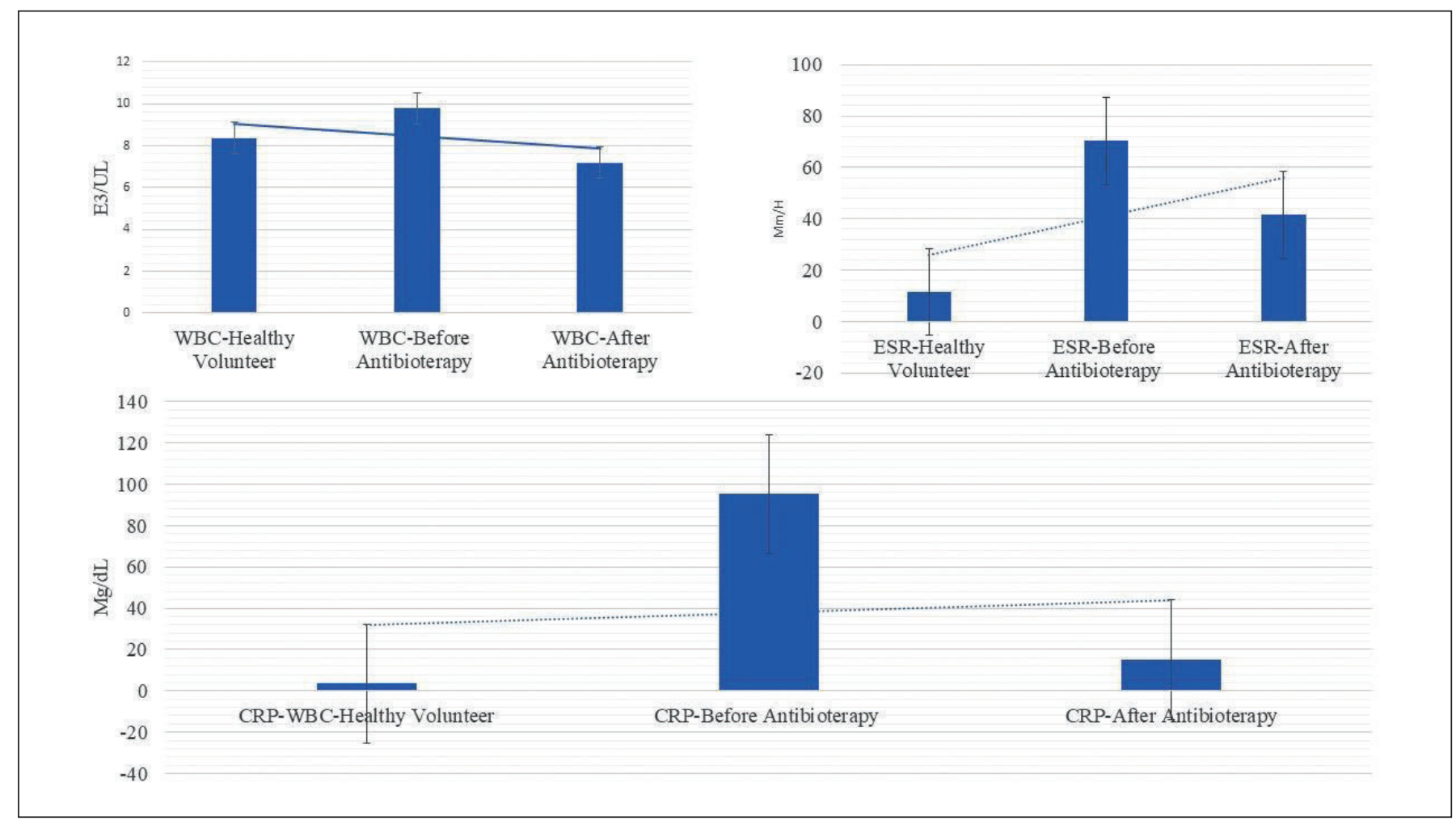

Figure 2: Data of the cases and volunteers in the comparison of infectious markers before and after treatment. 
culture antibiograms. It was also reported that the patients with spondylodiscitis responded to empirical antibiotic therapy and that age was not a predictive issue (5). It was emphasized that blood and tissue culture samples should be taken before starting the empirical antibiotic treatment of patients diagnosed with PVO. They concluded that effective and prompt empirical antibiotherapy should be implemented without delay until the culture results were obtained and that early surgical decompression might have a beneficial effect on the prognosis of the cases (5). In addition, it was indicated that the empirical antibiotics (mean antibiotherapy 73.45 days) were used for 11 (47.82\%) patients in which no microorganisms had been found and that the specific antimicrobial treatment continued for at least six weeks (5). It was reported that the administered treatment comprised intravenous mono or combined antibiotic therapy for a mean period of four weeks. In addition, they reported that oral, mono or combined antibiotics were prescribed for a further six weeks after the clinical symptoms regressed (5).

In the present study, diagnostic spinal MRI imaging was performed for the patients with culture-negative PVO $(n=8)$, who were examined retrospectively. The mean duration of intravenous antibiotherapy administered to the patients was $48.63 \pm 36.18$ (mean \pm SD) days and the shortest duration of treatment was four weeks. The patients received antimicrobial therapy either intravenously, mono or combined during the hospitalization period from the beginning of the diagnosis. After they were discharged, oral antibiotics having amoxicillin-clavulanic acid, ciprofloxacin, rifampicin, linezolid, or clindamycin as the active substance were prescribed. The administered pharmaceuticals were antistaphylococcal, bone and tissue perfusion-resistant antibiotics.

In their research, Pola et al. evaluated the studies performed in the last 20 years on pyogenic spondylodiscitis (18). They indicated that empirical antibiotic treatment when waiting for the results of cultures or in culture-negative cases should comprise broad-spectrum agents in association with agents active on Staphylococcus aureus. Furthermore, it was emphasized that the antibiotics active on methicillin-resistant Staphylococcus aureus should be included in treatment planning, based on local epidemiological data and that the antibiotherapy should not be less than four weeks (18).

In addition, Pola et al. reported that rifampicin was widely used in the empirical treatment of spondylodiscitis cases, but that no controlled trials were available to evaluate whether this combination was the right treatment option $(12,18)$.

In a 10-year retrospective cohort study conducted by Chong et al., it was found that of 129 cases with PVO, no causative organism was identified in $21.7 \%$ of the patients, and that open biopsy of vertebral tissue had a higher diagnostic yield than the other ones (7). They reported that only $15 \%$ of the patients had complete recovery after receiving a median of six-week intravenous antibiotic therapy. It was also reported that the oral antibiotic treatment continued for a median of 42 days after intravenous therapy. However, they concluded that the clinical outcomes for the patients with PVO were poor despite the empirical antibiotic treatment (7).
It is known that the ability of different antibiotics to penetrate bone may also vary (12). Fluoroquinolones, clindamycin, rifampicin, fusidic acid, and metronidazole have good levels of penetration into bone tissue. However, beta-lactam antibiotics and glycopeptides can reach moderate levels, and the bone tissue penetration of aminoglycoside group pharmaceuticals remains insufficient $(15,17)$.

In the literature, some studies suggest that broad-spectrum antibiotics with anti-staphylococcal activity should be recommended in patients in which PVO is suspected, considering the possible causative microorganisms $(9,15,17)$. While methicillin resistance is common in nosocomial-acquired Staphylococcus aureus strains, this resistance is less common in community-acquired strains. A combination of ciprofloxacin and clindamycin, which provides both staphylococcal and Gram-negative activity, good bone and disc penetration, and allows early access to oral treatment, is recommended as an empirical therapy $(9,15)$. In another study, the efficacy of the levofloxacin and rifampicin combination was found to be $78 \%$, while the efficacy of the amoxicillin/clavulanic acid and ciprofloxacin combination was found to be $82 \%$ in the empirical antibiotic therapy of PVO (17).

Adam et al. evaluated the efficacy of empirical antibiotic therapy in cases of spondylodiscitis (1). They planned to administer the following treatment to the patients: sulfamethoxazoletrimethoprim in combination with ciprofloxacin, sulfamethoxazole-trimethoprim in combination with ceftazidime, rifampicin in combination with levofloxacin, and imipenem in combination with cefoperazone sulbactam (1). It was emphasized that the empirical antibiotics to be administered should include antistaphylococcal activity (1). It was reported that the treatment process of the patients with spondylodiscitis who had histories of smoking-related bronchitis $(n=6)$, diabetes mellitus (5), and chronic alcoholism $(n=4)$ was more challenging. They also found that the main laboratory analyses were ESR, CPR, and WBC and that when readmitted, only one patient had a normal value of ESR. In their study, they observed that five patients were unresponsive to empirical therapy and that antibiotherapy should last from four to six months in patients with spondylodiscitis (1).

Cordero-Delgado et al. systematically evaluated the articles in which pyogenic spondylodiscitis cases were examined (8). They emphasized the importance of the epidemiology of etiologic agents and the need to pay attention to the ability of antibiotics to penetrate the bone tissue and intervertebral disc when planning antibiotic treatment. More importantly, following the systematic review of 642 studies (8), they concluded that there was not enough information concerning the use of empiric antibiotics in pyogenic spondylodiscitis and that the information on this subject was insufficient in the literature.

Unlike Pola et al. (18); Cordero-Delgado et al., emphasized that fluoroquinolones should be used in combination with rifampicin in the empirical treatment of pyogenic spondylodiscitis and that a pharmacological agent with vancomycin as the active ingredient should be added to this combination in the treatment of methicillin-resistant strains (8). 
In a study conducted by $Y u$ et al. (21), it was suggested that vancomycin and third- or fourth-generation cephalosporins should be preferred as empirical antibiotics in spondylodiscitis cases where the causative microorganism could not be detected. It was also suggested that broad-spectrum antibiotherapy should be preferred, even if empirically, because of the possibility of polymicrobial or multiple drug resistant organisms (21).

After examining the culture antibiogram results of the cases evaluated in our retrospective study, we found that the CRP parameter was statistically significant $(p<0.05)$ when evaluating the results of the treatment of the spondylodiscitis without proliferated microorganism, but the other parameters were not significant $(p>0.05)$.

Preferred antibacterial pharmacological agents include: a beta-lactam antibiotic and first-generation cephalosporin with bactericidal activity, an inhibitor of bacterial beta-lactamase, in a class of medications called ampicillin-like antibiotics, quinolone group, the combination of a fourth-generation extended-spectrum penicillin and a beta-lactamase inhibitor, an oxazolidinone class synthetic antibacterial antibiotic, and inhibits bacterial protein synthesis oxazolidinone class synthetic antibacterial antibiotic, inhibits bacterial protein synthesis by binding to bacterial $50 \mathrm{~S}$ ribosomal subunits.

Only one patient was found to have bactericidal semi-synthetic antibiotics in a combination of antibiotherapy.

Having observed that the clinical and laboratory response to the initial medical treatment could not be achieved at the desired level and that the radiological progression was detected despite this treatment modality, it was thought that different antibiotherapy options, including various preparations, should beused.

In addition to the spondylodiscitis, some patients were observed to have risk factors such as alcohol consumption $(n=1)$ and smoking $(n=1)$, and some patients had co-morbid diseases such as diabetes mellitus $(n=2)$ and arterial hypertension $(\mathrm{n}=2)$.

Major laboratory markers of the evaluated cases $(n=8)$ were ESR, CPR, and WBC (<11.4 e3 / ul). While the WBC values of all the patients before and after the treatment were in the normal range, the ESR values were decreased and they did not return to their normal range (Male $>5 \mathrm{~mm} / \mathrm{H}$; Female $>8$ $\mathrm{mm} / \mathrm{H})$. It was observed that the CRP values decreased to normal range $(<5 \mathrm{mg} / \mathrm{dL})$, except for two cases.

In this study, the number of cases in the study group $(n=24)$ was reduced $(n=8)$ based on the research criteria. While this might seem to be a limitation of the study, it is not a real limitation since the rate of spondylodiscitis is 2.4 per 100.000 (22). The real limitation of this study is its retrospective design.

\section{CONCLUSION}

There is a need for controlled, randomized clinical trials that include larger series to determine the appropriate empirical antibiotic regimen and duration of treatment of culturenegative PVO. There is also a need to increase awareness about using antibiotics correctly.

\section{REFERENCES}

1. Adam D, Papacocea T, Hornea I, Croitoru R: Postoperative spondylodiscitis. A review of 24 consecutive patients. Chirurgia (Bucur) 109: 90-94, 2014

2. Allard J, Carthey J, Cope J, Pitt M, Woodward S: Medication errors: Causes, prevention and reduction. $\mathrm{Br} \mathrm{J}$ Haematol 116: 255-265, 2002

3. Amin MEK, Amine A, Newegy MS: Perspectives of pharmacy staff on dispensing subtherapeutic doses of antibiotics: Atheory informed qualitative study. Int J Clin Pharm 39: 11101118, 2017

4. Beronius M, Bergman B, Anderson R: Vertebral osteomyelitis in Göteborg, Sweden: Aretrospective study of patients during 1990-95. Scan J Infect Dis 33: 527-532, 2001

5. Cervan AM, Colmenero Jde D, Del Arco A, Villanueva F, Guerado E: Spondylodiscitis in patients under haemodyalisis. Int Orthop 36: 421-426, 2012

6. Chelsom J, Solberg CO: Vertebral osteomyelitis at a Norwegian university hospital 1987-97: Clinical features, laboratory findings and outcome. Scand J Infect Dis 30: 147-151, 1998

7. Chong BSW, Brereton CJ,Gordon A, Davis JS: Epidemiology, microbiological diagnosis, and clinical outcomes in pyogenic vertebral osteomyelitis: A 10-year retrospective cohort study. Open Forum Infect Dis (serial online) 5: ofy037, 2018

8. Cordero-Delgado DA, Moheno-Gallardo AJ, Torres-González R, Mata-Hernández A, Elizalde-Martínez E, Pérez-Atanasio JM: Evidence and recommendation of empirical antimicrobial treatment in pyogenic spondylodiscitis: Systematic review. Rev Med Inst Mex Seguro Soc 55:6-13, 2017

9. Cottle L, Riordan T: Infectious spondylodiscitis. J Infect 56: 401-412, 2008

10. Dogan M, Mutlu LC, Yilmaz I, Bilir B, Varol Saracoglu G, Yildirim Guzelant A: Are treatment guides and rational drug use policies adequately exploited in combating respiratory system diseases? J Infect Public Health 9:42-51, 2016

11. File TM Jr: Duration and cessation of antimicrobial treatment. J Hosp Med 7:22-33, 2012

12. Karaarslan N, Yilmaz I, Ozbek H, Gurbuz MS, Oznam K, Ates $\mathrm{O}$, Erdem I: Is implant washing and wound irrigation with rifampicin effective for preventing surgical site infections in lumbar instrumentation? Turk Neurosurg 28(6):904-909, 2018

13. Kim DM, Kim SW: Delayed spondylodiscitis presenting as a skip lesion. Korean J Spine 14:14-16, 2017

14. Kim J, Kim YS, Peck KR, Kim ES, Cho SY, Ha YE, Kang CI, Chung DR, Song JH: Outcome of culture-negative pyogenic vertebral osteomyelitis: Comparison with microbiologically confirmed pyogenic vertebral osteomyelitis. Semin Arthritis Rheum 44: 246-252, 2014

15. Kwon JW, Hyun SJ, Han SH, Kim KJ, Jahng TA: Pyogenic vertebral osteomyelitis: Clinical features, diagnosis, and treatment. Korean J Spine 14: 27-34, 2017

16. Lemaignen A, Ghout I, Dinh A, Gras G, Fantin B, Zarrouk V, Carlier R, Loret JE, Denes E, Greder A, Lescure FX, Boutoille D, Tattevin P, Issartel B, Cottier JP, Bernard L: DTS (Duration of Treatment for Spondylodiscitis) Study Group: Characteristics of and risk factors for severe neurological deficit in patients with pyogenic vertebral osteomyelitis: A case-control study. Medicine (Baltimore) 96:e6387, 2017 
17. Luzzati R, Giacomazzi D: The empirical antibiotic therapy of pyogenic vertebral osteomyelitis. Semin Arthritis Rheum 41: e9, 2012

18. Pola E, Logroscino CA, Gentiempo M, Colangelo D, Mazzotta V, Di Meco E, Fantoni M: Medical and surgical treatment of pyogenic spondylodiscitis. Eur Rev Med Pharmacol Sci 16:35-49, 2012

19. Pola E, Taccari F, Autore G, Giovannenze F, Pambianco V, Cauda R, Maccauro G, Fantoni M: Multidisciplinary management of pyogenic spondylodiscitis: Epidemiological and clinical features, prognostic factors and long-term outcomes in 207 patients. Eur Spine J 27: 229-236, 2018
20. World Health Organization (WHO): The rational use of drugs. Report of the Conference of Experts. Nairobi, 1985:10-38. http://apps.who.int/medicinedocs/documents/s17054e/ s17054e.pdf Accessed on 18.09.2018

21. Yu SH, Kim DH, Kim HS, Nam KH, Choi BK, Han IH: Infectious spondylodiscitis by uncommon pathogens: A pitfall of empirical antibiotics. Korean J Spine 13:97-101, 2016

22. Zimmerly W: Vertebral osteomyelitis. N Eng J M 362:10221029, 2010 